\title{
The HPS Educational Paradigm Hierarchy Model in Physics Experiment Education of Military Academies
}

\author{
Rui Yang ${ }^{1}$ \\ Academy of Army Armored Force, Department of Physics and Chemistry \\ Beijing 100072
}

\begin{abstract}
HPS educational paradigm emphasizes the interactive relationship among science, technology and society, and has been concerned by many developed countries for a long time. Applying the HPS educational paradigm hierarchy model, namely the "basic principles level-history of science level" - STS level" with strong operability, to the physics experiment education in military academies, the shortcomings of traditional science education could be overcome.
\end{abstract}

Keywords-HPS education; Physics experiment teaching of university; Higher education of military academy

\section{INTRODUCTION}

HPS educational paradigm is a popular program for international science education reform, which also is paid more attention by China's educational and academic communities. In concerning of deficiencies in current physics experiment education of military academy, this article analyzes the necessity of introducing the HPS educational paradigm into the physics experiment courses of the military academy and puts forward the HPS educational paradigm hierarchy model with clear operability.

\section{OUTLINING HPS EDUCATIONAL PARADIGM}

The crucial purpose of HPS educational paradigm is to understand the nature and methods of science in the context of the history of science, philosophy of science, and sociology of science. HPS then is the abbreviation for two meanings: History and Philosophy of Science, or History, Philosophy and Sociology of science, [1]17 -22 which focuses on the nature, methods, development model, and significance of science.

History of science and philosophy of science accompany the long historical development of science, the two are closely related to each other. "Philosophy of science without history of science is empty, history of science without philosophy of science is blind." [2]102 The history of science and philosophy of science initiated as independent disciplines back in 1920s, the early representatives were George Sarton for the former and the school of logical positivism for the latter. In late 20th century, with the historicism and constructivism turns in the philosophy of science, a broader discipline was gradually formed, including disciplines such as the history of science, philosophy of science, history of technology, philosophy of technology, sociology of science, and sociology of scientific knowledge, that is, the STS (science, technology and society).
STS represents the contemporary spirit of modern society, which is not only evaluated important applications of science and technology, but also guards against and reflects on the undesirable consequences on human society and nature. STS comprehensives science as a social institution with sociological methods, places science in the process of society, and accomplish the disenchantment of science. STS, for scientists is the supplement to scientific research that provides norms and descriptions. For public, STS provides a reliable way to understand science as a layman of it.

HPS research is a part of the STS research. What is science, in the perspective of HPS, is more integrative detailed than the traditional view of science in (1) scientific knowledge is based on experience (positivism); (2) theory is temporarily accepted if it was not falsified yet (falsificationism); (3) scientific theory loaded with value and there is no such thing as absolute objective knowledge; (4) scientific knowledge rooted in social and cultural backgrounds, no universal scientific method. Science is not a completely objective and positive activity, rather it is essentially subjective and relativistic. Science can not only bring benefits to mankind, but also lead to social and natural disasters.

Traditional science education focuses only on the dissemination of scientific empirical knowledge for training engineers and scientists who have to master these knowledge and skills. Elites growing up under this paradigm, because of lack of comprehension of the complex relationship between science, technology and society, are more likely to make science and technology lead to negative consequences. On the other hand, science and technology are no longer the personal interests of scientists and engineers, they need to be understood, supported, and supervised by the public. Improving the scientific literacy of the public is not only a requirement for social progress, but also helps promote the development of science and technology. Therefore, western countries had successively promoted HPS educational paradigm since the 1980 s, with the purpose of populate people with the nature, culture and values of science. This new scientific education paradigm emphasizes the significance of science to society, culture, and humanity, not just the dissemination of specific scientific knowledge. 


\section{THE NECESSITIES OF INTRODUCING HPS EDUCATIONAL PARADIGM INTO PHYSICS EXPERIMENT TEACHING IN MILITARY ACADEMY}

The ideas and goals of the HPS educational paradigm are of great significance in contemporary society because natural science and its technological applications are more and more important in current age. [3]83-87 HPS educational paradigm not only provides educational ideas and concepts that are consistent with the spirit of the time, providing a comprehensive understanding of the positive and negative effects of science and technology. It also creates a good atmosphere for the development of science and technology by promoting the understanding of science of the public.

Certain applications of science and technology will lead to far-reaching and even catastrophic consequences, some of which are initially unknown, such as the widespread use of DDT leading to ecological disasters; some are intentional, such as the enormous destructive power of nuclear weapons. The HPS study emphasizes symmetrically focusing on the possible consequences of science and technology, that is, not only advocates the positive value of science and technology, but also emphasizes vigilance and prevention of their possible negative value. In such manner, for those who will become scientists and engineers in the future, the case of scientific history and the philosophy of science will provide them with normative values for realizing the positive consequences of science and technology; for other public, HPS education makes them properly understand and appreciate science and technology, to eliminate the panic or anxiety caused by the lack of understanding. Then the public could participate in the promotion of benign consensus for developing science and technology, without blind obsession or implicit refusion. In the era of "big science" today, breakthroughs of frontal scientific research require a lot of resources, if there is no support and appreciation of the public, such scientific research activities will be difficult to sustain.

Reforming science courses and training science teachers are two main approaches for implementing the HPS educational paradigm, which usually managed by official education departments, such as formulating HPS educational plans and decrees, adding or supplementing HPS related courses, improving science teachers' knowledge and teaching skills concerning HPS, etc. In China, HPS education research is still in development, but on the operational level, the HPS educational paradigm can be implemented by science teachers, without changing the whole picture of current education.

For higher education, the introduction of HPS education is necessary. [4] 103-108 HPS educational paradigm can (1) directly supplement and promote the teaching of scientific knowledge and professional skills. By revealing the evolution of science and technology in history, rather than merely disseminating specific knowledge, students' understanding of scientific knowledge and the essence of science would be intensified, and their practical ability in scientific research and problem solving would be cultivated; (2) cultivate students effectively of scientific spirits, which including rational spirit of seeking and exploring, the empirical spirit of experimental verification, the critical spirit of refusing to follow the authority blindly, and the innovated spirit of continuous promotion. "Long-term and rigorous scientific training is the indispensable way to cultivate the scientific spirit", [5]21 and reflections on and references to previous experiences in the history of science are necessary supplements; (3) cultivate students' awareness of social responsibility by emphasizing the history of science and technology as the warning and enlightenment, this would make students more accurately grasp the connotation of scientific norms and scientific spirit, profoundly understand the humanistic implications of science and technology, enhance their awareness of social responsibility and their sense of historical mission, and establish their professional ethics for benefiting mankind with appropriate applications of science and technology.

Besides necessities mentioned above, for introducing the HPS educational paradigm into physics experiment teaching in the military academy, there are two supplements as following:

(1) Talents cultivated by military academies will become the backbone of the people's army in the future. HPS education could be fostering their scientific spirit, which would promote their behavior of seeking truth from facts and better serving the national defense career. This is also the significance of HPS educational paradigm for physics experiment teaching of military academies.

(2) Science and technology have played an important role in national defense. For applying science and technology properly in the national defense industry, it requires a longterm vision for national defense personnel, comprehending the interactive relationships of science, technology and society, this is just one main goal of HPS educational paradigm.

\section{THE HPS EdUCATIONAL PARAdigM HIERARCHY MODEL}

There is no fundamental conflict between the HPS educational paradigm and the traditional science education paradigm, and the dissemination of scientific knowledge is the same basis for them. On this basis, it is possible to propose the HPS educational paradigm hierarchy model with clear operationality, which will infiltrate HPS education concepts and related knowledge into traditional science education and implement HPS educational paradigm without changing the overall structure of education system.

Hall Effect experiment, for instance, the experiment teaching of which is divided into three parts, (1) explaining concerned principles: the concept and derivation of the Hall Effect; (2) explaining the experiment request: targets, devices and operations of the experiment; (3) operating the experiment by students: record and process experimental data to obtain experimental conclusions.

The first two parts determine whether students would understand and accept experimental principles, which is the basis of this experimental teaching. However, in traditional science education, science teachers are often confined to explaining specific formulas and calculations, while ignoring the history of discovering the Hall Effect, neither the actual applications, nor possible practical consequences. Then, on the one hand, this principle becomes a rigid theory to students 
which is mostly irrelevant with the reality; on the other hand, the experiment teaching is difficult to effectively cultivate students' scientific research ability and scientific spirit. The state of student learning is often passive, they are inclined to be satisfied with the basic requirements of experimental teaching objectives, that is to complete the experiment and finish the experimental report. This kind of teaching can hardly make students profoundly enlightened, nor attracting students' interest in learning.

Implementing the HPS educational paradigm can improve the above situation. However, in the practical situation of China, it would not be easily reform the whole structure of educational system, therefore, the Hierarchy Model proposed below is valued, which can effectively implement HPS educational paradigm without changing the current system of curriculum. The crucial part of above picture is the science teachers. Teachers are representatives and direct practitioners of HPS educational paradigm, therefore it is important that science teachers should know enough HPS knowledge and ideas, and directly enlighten students with appropriate comprehensions of science, technology and their relationships with society. Science teachers should actively strengthen their knowledge of HPS background because their initiatives would directly determine whether the HPS educational paradigm can practically and effectively penetrate the usual science education curriculum.

In view of the deficiencies in the usual physics experiment teaching in military academies and universities, the traditional teaching mentioned above should be extended as the following hierarchy model with permeation of the HPS educational paradigm:

(1) The basic principle level: the principle, concept and derivation of the Hall Effect;

(2) The history of science level: a brief history of discovering the Hall Effect, further development of applications, such as the quantum Hall Effect.

(3) The STS level: based on the characteristics of students in military academies, examples are given of real applications of Hall Effect in military technology.

The basic principle level among the three levels is the fundamental part, which bears the function of traditional science education to disseminate specific scientific knowledge. The history of science level undertakes the function of cultivating students' scientific spirit and scientific methodology The STS level oriented with pragmatism, that is, associating the theoretic knowledge with the practical national defense career of military academy students and cultivate their social responsibility to properly develop and use science and technology to serve the national defense industry. The three levels are related to each other progressively, the foundation is disseminating scientific knowledge, the approach is HPS educational paradigm, and ultimately purpose is to stimulating students' enthusiasm for learning and cultivating their sense of responsibility. Among the three, the third level is of significance in practice, therefore, teachers are required to pay more attention to it in order to developing students' proper value orientation and form the active sense of social responsibility.

To sum up, the HPS educational paradigm hierarchy model improves the content of traditional physics experiment teaching, and for educational audiences with different characteristics, the history of science level and the STS level should be given corresponding emphasis. For military school students, the development and application of science and technology in national defense are both in line with their professional orientation and closely related to their national defense career.

\section{CONCLUDING REMARKS}

Based on the dissemination of specific scientific knowledge the HPS educational paradigm emphasizes the value of science and its application to society, culture, and humanity. The advantage of HPS education over traditional science education is that it is more likely to motivate students' enthusiasm and interest in learning, and it is more likely to cultivate sci-tech talents with a comprehensive and long-term vision.

For physics experiment teaching in military academies, the penetration of HPS educational paradigm is necessary. The HPS education hierarchy model has operability and practical significance. Without radically changing the structure of the curriculum, it is emphasized that science teachers should actively implement the concept of HPS education. This would improve the deficiencies of traditional physics experiment teaching in military science education, and truly cultivate national defense talent with scientific spirit and social responsibility.

\section{ACKNOWLEDGMENT}

${ }^{1}$ Yang Rui (1986 ) is the physics lecturer at Academy of Army Armored Force. Email: 376224850@qq.com

\section{REFERENCES}

[1] WANG Xiao-hong, YUE Peng-fei, FU Jing-hui. The Origination of HPS in US and It's Contributions to Methodology of Scientific Discovery [J]. Studies in Dialectics of Nature, 2014, 30(03):17-22.

[2] Lakatos I. The methodology of scientific research programmes: Philosophical papers Vol. 1 [M]. Cambridge University Press, 1978.

[3] ZHANG Jing. HPS (History, Philosophy and Sociology of Science): A New Paradigm of Scientific Education [J]. Studies in Dialectics of Nature, 2008(09):83-87.

[4] WU Zhi-yuan. An Idea of Leading the HPS Education into the University Teaching: From the Angle of View of the Quality Education [J]. , 2009, 25(05):103-108.

[5] LIU Da-chun. The outline of dialectics of nature. Second Edition [M] China Renmin University Press, 2008. 